\title{
THE OCEANS AND THE EARTH'S ROTATION
}

\author{
P. Brosche \\ Observatium Hoher List \\ de Universitäts-Sternwarte Bonn \\ D-5568 Daun
}

F.R. Germany

\begin{abstract}
In the long run, the tidal interaction between the Moon and the solid Earth is mediated by the oceans. It produces the retardation of the Earth's rotation known as 'tidal friction'. Due to the changing configuration of the continents, it is a non-monotonic function of time. Tides of the solid Earth dominate the short-periodic tidal effects while the exchange with the atmosphere is preponderant in climatic changes, especially with an annual signature. It is shown that the influences of the oceans within such short time-scales must be taken into account for tidal and for non-tidal variations as well if one wants to model the Earth's rotation at the $\mathrm{cm}$-level corresponding to the most advanced observational techniques.
\end{abstract}

\section{INTRODUCTION}

Two modern means of observation have stimulated our topic in the recent decades: radio interferometry and laser ranging. With regard to these precise techniques, the geophysics needed for interpretation often seems to consist of understanding the solid Earth and the atmosphere, while influences of the oceans are often neglected. The aim of this paper is a warning "Yes, but don't take it for the whole truth". There are two broad reasons for this warning: (a) The time scales covered by the new techniques (at most a few decades) are only a small window of the whole spectrum of Earth's rotation. And although it is natural to concentrate on the best observed part, it would not be wise to forget completely the other parts. For practical purposes the present measurements are of greatest value, but the scientific value of variations at longer time-scales may be of the same order. They give us also a greater chance to observe extreme variations of the same kind as are observed at shorter time-scales. The extreme cases have the advantage that they may help us unravel the intricate network of causal relations in a simplified version. (b) The oceans can cause specific variations of the Earth's rotation which the other constituents cannot. This is most obvious for the tides, where the oceans have a greater mobility than the solid parts but a greater regularity of motion than the atmosphere.

In what follows we shall give a small number of characteristic examples, sorted by the character of the interaction into tidal and non-tidal ones. 


\section{TIDAL EFFECTS}

Tides of the solid Earth act on the Earth's rotation only via the influence on the moments of inertia and these moments do not measurably depend on the specific relative position of the Earth's surface features with respect to the disturbing body. Hence the $\mathrm{M}_{2}$ tidal forces acting on the solid Earth cause a certain constant change in the axial moment of inertia. In contrast, in the oceans, the $\mathrm{M}_{2}$ tide (a) produces a varying moment of inertia $\theta(\tau)$, and (b) transfers varying amounts of angular momentun $P_{m}$ into the motions of the water. Both components together cause a sinusoidal variation of the Earth's angular velocity $\omega$ (if it is the solid Earth which has to fulfill the conservation of angular momentum in the whole Earth system). By integrating $\omega$ in time, we obtain a contribution to UT varying sinusoidally with $\mathrm{M}_{2}$ at phase $\tau$. The numbers are as follows (Baader et al.,1983):

$$
\begin{gathered}
\Delta \theta(\tau)=9.5 \times 10^{28} \sin \left(\tau+41^{\circ} .4\right) \mathrm{kg} \mathrm{m}^{2} \\
\Delta \mathrm{P}_{\mathrm{m}}(\tau)=24.6 \times 10^{24} \sin \left(\tau-189^{\circ} .5\right) \mathrm{kg} \mathrm{m}^{2} \mathrm{~s}^{-1} \\
\Delta \omega(\tau)=26.0 \times 10^{-14} \sin \left(\tau-24^{\circ} .4\right) \mathrm{s}^{-1} \\
\Delta \mathrm{UT}(\tau)=0.025 \sin \left(\tau+245^{\circ} .6\right) \mathrm{ms}
\end{gathered}
$$

This effect is admittedly small, but there is no such effect from the solid Earth and, therefore, it should be readily detected as soon as the measurement accuracy allows it. From an incomplete model of the oceanic $K_{1}$ tide we find $\theta$ variations of the same order of magnitude. With the longer integration time - twice the period of $\mathbf{M}_{2}$ - we expect an even larger effect in UT. In general, we expect a maximum influence on UT in the case of fortnightly tides because of the longer integration time and still considerable tidal force components. These effects illustrate also the necessity for measurement at short time intervals if one is concerned with $\mathrm{cm}$ level accuracy.

The large phase lag of oceanic tides (in comparison with solid Earth tides) is the reason for the dominance of this kind of tidal effect in the long run: the secular effect, the tidal friction in the Earth-Moon system, is almost exclusively mediated by the oceans. The average tidal torque is today $\mathrm{L}=5 \times 10^{16} \mathrm{~N} \cdot \mathrm{m}$, which corresponds to a retardation of the Earth's angular velocity

$$
\dot{\omega}=-5 \times 10^{22} \mathrm{rad} \cdot \mathrm{s}^{-2}
$$

or a decrease in the Earth's rotational energy of about $1 / 10$ of the radioactive heat production in the Earth's interior:

$$
d \mathrm{E}_{\mathrm{rot}} / d t=-4 \mathrm{TW}
$$

It should be emphasized also, however, that this effect is not a dull, uniform change but instead has a pronounced time structure: in only 20 million years, continental drift can change the delicate resonance structure of the oceans in such a way that the torque varies by a factor of two (Brosche and Hoevel, 1983). Naturally, this occurs also at time scales of 100-1000 million years (Gotlib and Kagan, 1985). 


\section{NON-TIDAL EFFECTS}

While for the tidal effects we had to look also at the solid Earth, here we have to regard the atmosphere. First of all, most of the short time variation of the Earth's rotation has been found to 'mirror' the angular momentum content of the atmosphere (Hide, 1984) as it should if angular momentum is conserved. The departures from strict conservation are of the order of $10 \%$ of the atmospheric variation and the estimated influence of the oceans is of the same order. It is a matter of personal viewpoint whether or not one should start oceanographic investigations at this stage. I would say, it is time to begin if we want to do $\mathrm{cm}$-level geodynamics. There is general agreement that the first oceanic candidate object is the Antarctic Circumpolar Current (ACC). The only discrepancy in the angular momentum balance of solid Earth plus atmosphere (and that marginal) was found in the semiannual term (about $50 \mu \mathrm{s}$ in the length of the day, Eubanks et al., 1984). We have shown that this defect can be understood in amplitude and phase by an intermediate storage of angular momentum in the ACC: the antarctic storms drive the ACC and after about 40 days the water transfers the angular momentum to the solid Earth (Brosche and Sündermann, 1985). This again shows that the oceanic contribution cannot be neglected if we are aiming at cm-level accuracy.

Finally, I would like to comment on the change $\Delta \mathrm{LOD}=-1.0 \mathrm{~ms} /$ century in the Earth's rotation around A.D. 1000, found by Stephenson and Morrison (1984). A change in average wind speed of $1 \mathrm{~m} / \mathrm{s}$ could have been the cause, but this is meteorologically an implausibly high value (Hide, personal communication.) Instead, I find it appealing to associate this change with the warm era around A.D. 1000 known qualitatively from many sources. The Vikings were able to reach North America at that time because the climate of the northern Atlantic was not nearly as rough as today. The observed change could have been produced by a sea level increase of $20 \mathrm{~cm}$ which had to come from land ice. In the case of Antarctica, it would mean about a $6 \mathrm{~m}$ decrease of its ice. Sea level variations with time scales of one millenium are not easy to find. For the North Sea, they are established by the long tradition of building dykes. It seems that at least a relative variation of the desired magnitude is revealed by the data (Rohde 1978). 


\section{REFERENCES}

Baader, H.R., Brosche, P., Hoevel, W. 1983: J. Geophys 52, 140

Brosche, P., Hoevel, W. 1983: in "Tidal Friction and the Earth's Rotation II" (eds. P. Brosche and J. Sündermann) Springer, Berlin-Heidelberg-New York, p. 175

Brosche, P., Sündermann, J. 1985: Deutsche hydrographische Zeitschrift 98,1

Eubanks, T.M., Dickey, J.O., Steppe, J.A., 1984: JPL Geod. Geophys. Prepr. No. 106

Gotlib, V.Yu., Kagan, B.A., 1985: Deutsche hydrographische Zeitschrift 98, 43

Hide, R., 1984: Phil. Trans.R. Soc. London A919, 107

Rohde, H., 1978: Die Küste, No. 32, p. 6

Stephenson, F.R., Morrison, L.V., 1984: Phil.Trans. R. Soc. London A319, 47

\section{DISCUSSION}

Rosen: With regard to the semi-annual discrepancy referred to by the author, it turns out that the discrepancy disappears if mid- and upper-stratospheric wind data are added to the routinely available atomospheric momentum series. This result was published by Rosen \& Salstein in Journal of Geophysical Research. Hence, there appears to be no need to turn to the Antarctic circumpolar current to cloee the momentum budget at this period. 